\title{
MR imaging findings in some rare neurological complications of paediatric cancer
}

\author{
Tetsuhiko Okabe ${ }^{1,2} \cdot$ Taiki Nozaki $^{1} \cdot$ Noriko Aida $^{3} \cdot$ Jay Starkey $^{1} \cdot$ Mikako Enokizono $^{3,4} \cdot$ Tetsu Niwa $^{3,5}$. \\ Atsuhiko Handa ${ }^{1,6}$ - Yuji Numaguchi ${ }^{1}$ - Yasuyuki Kurihara ${ }^{1}$
}

Received: 18 December 2017 /Revised: 6 April 2018 / Accepted: 11 April 2018 /Published online: 15 May 2018

(C) The Author(s) 2018

\begin{abstract}
Neurological complications of paediatric cancers are a substantial problem. Complications can be primary from central nervous system (CNS) spread or secondary from indirect or remote effects of cancer, as well as cancer treatments such as chemotherapy and radiation therapy. In this review, we present the clinical and imaging findings of rare but important neurological complications in paediatric patients with cancer. Neurological complications are classified into three phases: pre-treatment, treatment and post-remission. Paraneoplastic neurological syndromes, hyperviscosity syndrome, haemophagocytic lymphohistiocytosis and infection are found in the pre-treatment phase, while Trousseau's syndrome, posterior reversible encephalopathy syndrome and methotrexate neurotoxicity are found in the treatment phase; though some complications overlap between the pre-treatment and treatment phases. Hippocampal sclerosis, radiation induced tumour, radiation induced focal haemosiderin deposition and radiation-induced white matter injury are found in the post-remission phase. With increasingly long survival after treatment, CNS complications have become more common. It is critical for radiologists to recognise neurological complications related to paediatric cancer or treatment. Magnetic resonance imaging (MRI) plays a significant role in the recognition and proper management of the neurological complications of paediatric cancer.

\section{Teaching Points}

- Neurological complications of paediatric cancer include various entities.

- Neurological complications are classified into three phases: pre-treatment, treatment and post-remission.

- Radiologists should be familiar with clinical and imaging findings of neurological complications.

- MRI features may be characteristic and lead to early diagnosis and proper treatments.
\end{abstract}

Keywords Paediatric cancer $\cdot$ Treatment $\cdot$ Neurological complication $\cdot$ MRI, imaging

Tetsuhiko Okabe

hocoroco2013-study@yahoo.co.jp

1 Department of Radiology, St. Luke's International Hospital, 9-1 Akashi-cho, Chuo-ku, Tokyo 104-8560, Japan

2 Department of Radiology, Yokohama City University, Kanagawa, Japan

3 Department of Radiology, Kanagawa Children's Medical Center, Kanagawa, Japan

4 Department of Radiology, National Center of Neurology and Psychiatry, Tokyo, Japan

5 Department of Radiology, Tokai University School of Medicine, Kanagawa, Japan

6 MassGeneral Hospital for Children and Harvard Medical School, Boston, MA, USA

\section{Introduction}

Malignant tumours are the most common disease-related cause of death for the people under 20 years of age. With survival rates rapidly increasing with new treatments, currently two-thirds of children who suffer from paediatric cancers become long-term survivors. As survival rates for children with cancer have improved, so have the number of people who develop complications, with at least $70 \%$ of paediatric cancer survivors having some complication within 30 years from the onset their disease [1] (Table 1). Leukaemia and central nervous system (CNS) tumours make up the majority of paediatric malignancies. The most common types of brain tumours of children are astrocytoma, medulloblastoma and ependymoma [2]. Because current treatments for brain 
Table 1 Neurological complications associated with paediatric cancer

$\begin{array}{ll}\text { Pre-treatment phase } & \begin{array}{l}\text { Paraneoplastic neurological syndromes } \\ \text { Haperviscosity syndrome }\end{array} \\ & \text { Haemophagocytic lymphohistiocytosis } \\ & \text { Infection }^{\mathrm{a}} \\ \text { Treatment phase } & \text { Trousseau's syndrome }^{\mathrm{b}} \\ & \text { Posterior reversible encephalopathy syndrome } \\ & \text { Methotrexate neurotoxicity } \\ \text { Post-remission phase } & \text { Hippocampal sclerosis } \\ & \text { Radiation induced tumour } \\ & \text { Radiation induced focal haemosiderin deposition } \\ & \text { Radiation induced white matter injury }\end{array}$

${ }^{a}$ Sometimes seen in treatment phase

${ }^{\mathrm{b}}$ Sometimes seen in pre-treatment phase

tumours and leukaemia include intrathecal chemotherapy and cranial irradiation, which are potentially neurotoxic, neurological complications are often found in patients with paediatric cancer [3]. It is necessary to be familiar with the complications that can be seen on imaging related to cancers and their treatment, as imaging is essential for early diagnosis and proper treatment to minimise adverse effects. In this review, we present the clinical and imaging findings of rare but important neurological complications in paediatric patients with cancer in the pre-treatment, treatment and post-remission phases.

\section{Pre-treatment phase}

\section{Paraneoplastic neurological syndromes}

Paraneoplastic neurological syndromes are defined as neurological syndromes caused by an autoimmune mechanism in cancer patients. Diagnosis of a paraneoplastic syndrome in pre-treatment patients must exclude local neuropathy due to metastasis, opportunistic infections accompanying decreased immune response, vascular disorders with coagulopathy and neuropathy accompanied by malnutrition. It should be noted that paraneoplastic syndromes are also sometimes found in the treatment phase. Although paraneoplastic syndromes can affect any part of the neuraxis in children, the CNS is the most commonly affected [4].

The most common paraneoplastic syndromes in children are: (1) opsoclonus myoclonus syndrome (OMS), (2) limbic encephalitis, and (3) anti- $N$-methyl-D-aspartate (NMDA) receptor encephalitis [5]. Regarding OMS, neuroblastoma is found in as many as $50 \%$ of children with the disease, although OMS occurs in just 2-3\% of children with neuroblastoma [4]. The most frequently associated neoplasms with limbic encephalitis in children are neuroblastoma, Hodgkin lymphoma, ovarian teratoma and testicular tumour. Manifestation of paraneoplastic limbic encephalitis precedes the detection of cancer in $60 \%$ of patients [4]. Anti-NMDA receptor encephalitis is a paraneoplastic syndrome associated with teratomas. Most patients with anti-NMDA receptor encephalitis have full or substantial recovery after treatment. Although the most frequently associated neoplasm is ovarian teratoma, others such as mediastinal teratomas, testicular tumours and small cell lung cancers have been reported.

Typical features of limbic encephalitis include personality changes, irritability, seizures, cognitive dysfunction and memory deterioration. Children with anti-NMDA receptor encephalitis have behavioural or personality changes, sleep dysfunction, dyskinesia, dystonia and/or dysautonomia.

Magnetic resonance imaging (MRI) demonstrates high signal intensity on T2-weighted images in one or both mesial temporal lobes. MRI may have abnormal signal intensities in other regions, such as the brainstem, hypothalamus, thalamus and cingulate gyrus (Fig. 1) [6]. The diagnosis of paraneoplastic limbic encephalitis must be made comprehensively in combination with the imaging and clinical findings, including cerebrospinal fluid examination, because it is difficult to distinguish from other diseases such as herpes simplex encephalitis or convulsive encephalopathy which have similar imaging features. In anti-NMDA receptor-related encephalopathy, MRI may demonstrate hyperintensities in the medial temporal lobes, cortical and subcortical regions, basal ganglia, brain stem and cerebellum on fluid attenuated inversion recovery (FLAIR) images. These regions may also show contrast enhancement [7].

Treatment is usually with immunomodulators such as steroids or intravenous immunoglobulins and plasmapheresis, along with treatment of the primary tumour.

\section{Hyperviscosity syndrome (HVS)}

HVS has been recognised in patients with macroglobulinaemia, a type of non-Hodgkin lymphoma, since it was first described by Waldenstrom in 1944 [8]. When the serum viscosity rises, blood flow is delayed and peripheral circulation disturbance occurs. The classic triad for HVS consists of bleeding, neurological symptoms and visual disturbances. However, in addition to these symptoms, a variety of end organ damage can be observed.

Primary macroglobulinaemia is the most common cause of HVS, which accounts for 85-90\% of HVS. Multiple myeloma is the second leading cause overall. While multiple myeloma can occur in children, it is exceedingly rare. HVS can also be caused by leukaemia because hyperleukocytosis, which can lead to leukostasis, is often found in leukaemia. Hyperleukocytosis is found in 5-13\% patients with acute myeloid leukaemia and 10-30\% patients with acute lymphocytic leukaemia [9]. 
Fig. 1 Paraneoplastic limbic encephalitis. A 17-year-old boy with recurrent medulloblastoma presented with seizure and altered mental status. a FLAIR image shows hyperintensities in the mesial temporal regions (arrows) b Contrast enhanced fatsuppressed T1-weighted image shows nodular meningeal enhancements in the left Sylvian fissure (arrowheads), suggestive of recurrence
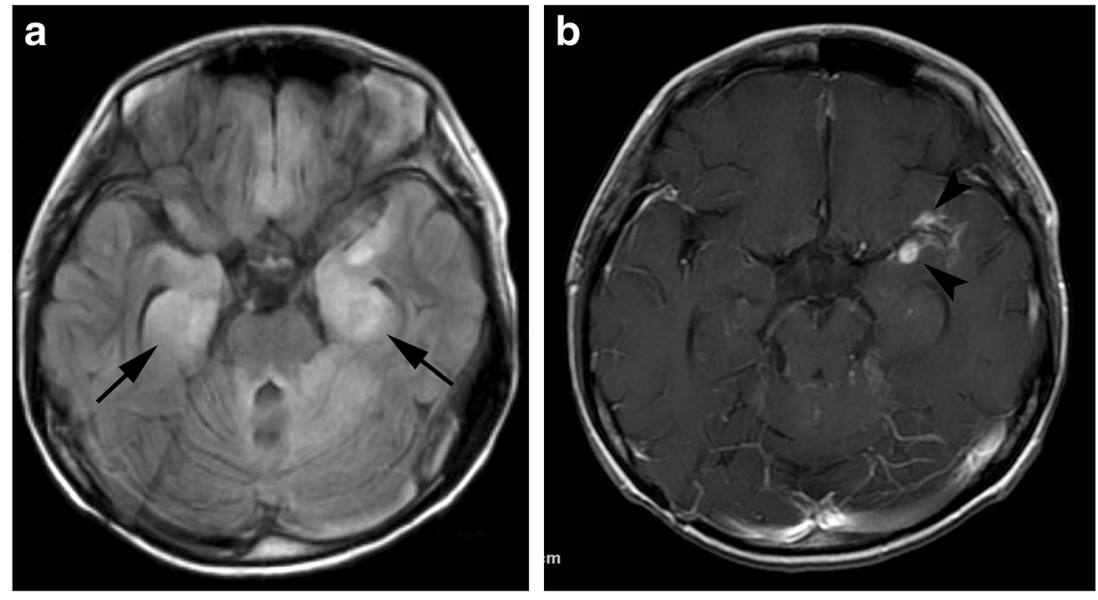

In patients with HVS, congestive heart failure, renal dysfunction, anorexia, fatigue and weakness may occur in addition to the classic triad. The most frequent symptom is bleeding, especially gingival and nasal. Although the number of platelets is usually near-normal, repetitive bleeding is characteristic. Bleeding may result from blood vessel wall abnormality caused by an intravascular friction phenomenon due to the increased blood viscosity. Neurological symptoms are common in hyperviscosity syndrome, including headache, dizziness, vision disorder, gait disturbance, sensory deafness, convulsions, coma and cerebral infarction. MRI may show multifocal parenchymal microhaemorrhages or frank haematoma formation [10, 11] (Fig. 2).

Plasma exchange therapy is widely used for the treatment of hyperviscosity syndrome, and clinical symptoms improve by decreasing blood viscosity [12]. In primary macroglobulinaemia and multiple myeloma, it is necessary to use chemotherapy in addition to plasma exchange.

\section{Haemophagocytic lymphohistiocytosis (HLH)}

HLH is a disease characterised by systemic proliferation of histiocytes. HLH is divided into the familial/primary and the secondary types.

In both primary and secondary HLH, cytokinaemia associated with excessive activation of NK cells and cytotoxic T cells and accompanying tissue damage is the hallmark of the disease. When severe, infiltration into the central nervous system occurs. Histopathological findings of CNS invasion of HLH are classified into stages I-III. Stage I primarily shows only leptomeningeal infiltrates of lymphocytes and histiocytes/macrophages. Stage II shows additional parenchymal involvement with perivascular infiltrations. Stage III reveals cerebral tissue necrosis and demyelination in addition to massive white matter infiltration [13]. Familial HLH is genetic, mainly due to abnormality of perforin, MUNC13-4, syntaxin or MUNC18-2 [14]. Secondary types are associated with malignancy, infection, autoimmune disease and drugs. Although the most common cause of malignancy associated HLH is lymphoma and leukaemia, malignant solid tumours also cause HLH $[15,16]$. Malignancy associated HLH is usually seen in the pre-treatment phase; however, it may also be seen in the treatment phase.

HLH is associated with fever, hepatosplenomegaly, lymphadenopathy, rash and bleeding tendency. Laboratory and pathological examinations reveal blood cell phagocytosis of bone marrow, pancytopenia, liver dysfunction, hypertriglyceridaemia, low fibrinogen plasma and high value of serum ferritin.

MRI include diffuse leptomeningeal and perivascular enhancement, which corresponds to meningeal and perivascular infiltrations of histiocytes and lymphocytes, patchy areas of an increased T2 signal intensity in the white matter of the both cerebral hemispheres, and diffuse cerebral and cerebellar parenchymal volume loss (Fig. 3). In some cases, nodular or ring enhancement of parenchymal lesions occurs due to the compromised blood-brain barrier in areas of active demyelination. Diffusion-weighted imaging (DWI) shows diffusion restriction in white matter lesions during the acute phase [17]. Differentiation from posterior reversible encephalopathy syndrome (PRES) is often a problem both clinically and radiologically.

Although treatments of HLH have not been established, initial goals of treatments in HLH have been to suppress the overactive immune system, thus preventing immunemediated organ damage. Patients with malignancy associated HLH require control of the HLH followed by treatment of the underlying malignancy.

\section{Infection}

Control of infection is central in the management of cancer. Even before treatment, paediatric patients with malignant tumours are prone to infection because of decreased granulocytes, mucositis and reduced mucociliary clearance 
Fig. 2 Hyperviscosity syndrome. A 10-year-old girl with acute lymphocytic leukaemia, whose white blood cell count reached 400,000 , presented with headache and vomiting. a A T1-weighted image shows hyperintensity in the pons and bilateral cerebellar hemispheres (arrows). b These lesions show hyperintensity with a hypointensity rim on $\mathrm{T} 2-$ weighted image (arrows), indicating haemorrhage in the subacute phase. c T2*-weighted image demonstrates a number of microhaemorrhages which are not seen on T1- and T2-weighted images
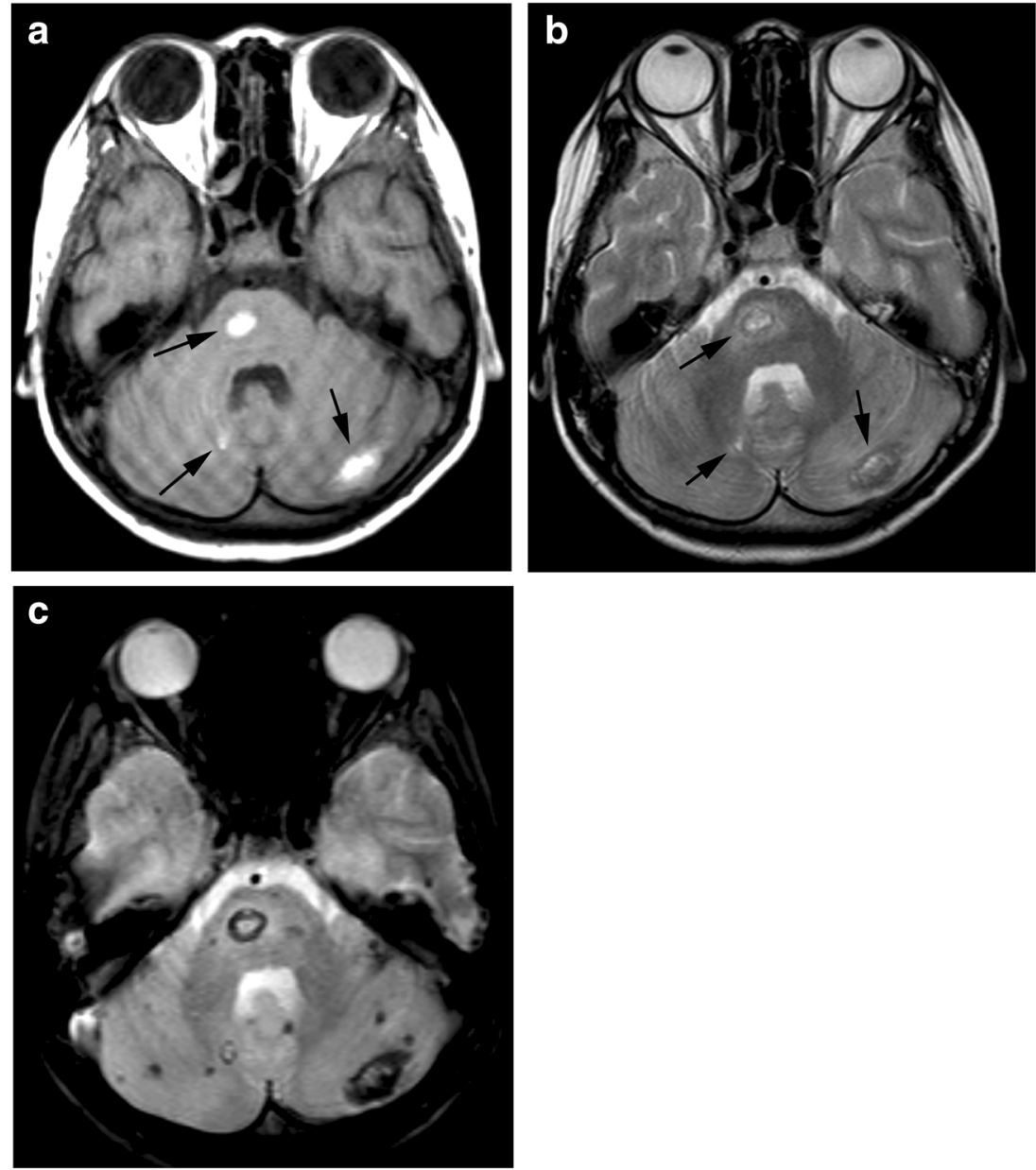

or depletion of physiological flora, together with immunosuppression due to primary disease.

Intracranial infection may result from direct spread from sinusitis or from haematogenous spread, especially in the form of septic emboli complicating endocarditis. Such infections may be fungal, bacterial or viral.

Fungal infection Fungal infection typically affects children having absolute granulocyte counts of less than $100 / \mathrm{mm}^{3}$ for longer than 2 weeks [18].

The MRI appearance varies with the causative agent. Aspergillus may cause an infectious vasculopathy, leading initially to acute multiple infarctions or haemorrhage and later to extension into surrounding tissue as an infectious cerebritis or occasionally evolving into an abscess [19]. Fungal abscesses may have central restricted diffusion because of proteinaceous fluid and cellular infiltration in the lesions [20]. Haemorrhage is found in $25 \%$ of patients [21]. The contrast enhancement of the lesion is strong in immunocompetent patients but is often characteristically weak in immunocompromised patients [19]. Typical sites of involvement in Aspergillus vasculopathy include the basal ganglia, thalami and corpus callosum, reflecting a predisposition to involve the perforating arteries, as well as the subcortical regions. Encasement of intracranial arteries and vasculitis is found on MR angiography. Candidiasis may cause numerous microabcesses at the greywhite matter junction, basal nuclei and cerebellum, while haemorrhage and infarction are relatively rare [22]. Formation of numerous abscesses can be seen in nocardiosis, resulting in hydrocephalus, epistaxis and meningitis.

Because fungal culture tests are time-consuming and often do not lead to definitive diagnosis, empirical antifungal treatments are recommended for high-risk patients.

Bacterial infection Although bacterial infections of the CNS are less common than fungal disease in immunocompromised patients with cancer, infection with Listeria monocytogenes and Bacillus cereus is well known [23, 24]. Clinical symptoms, cerebral spinal fluid (CSF) examination and laboratory data are important for the diagnosis of bacterial meningitis.

MRI is useful for detecting cerebral oedema, subdural effusions/abscess and arterial or venous infarction associated with meningitis. On MRI, hyperintensities are shown in the subarachnoid spaces on the FLAIR imaging, reflecting an increase in protein concentrations. Meningeal enhancement is observed on contrast MRI 
Fig. 3 Haemophagocytic lymphohistiocytosis. A 14-yearold girl with myelodysplastic syndrome presented with seizure. a A T2-weighted image shows patchy hyperintensities with swelling in the frontal and parietal lobes (arrows). b FLAIR image shows hyperintensity of these lesions (arrows). c T2*-weighted image shows a number of microhaemorrhages in these lesions (arrowheads). d Postcontrast image shows nodular enhancement along the leptmeninx (white arrows)
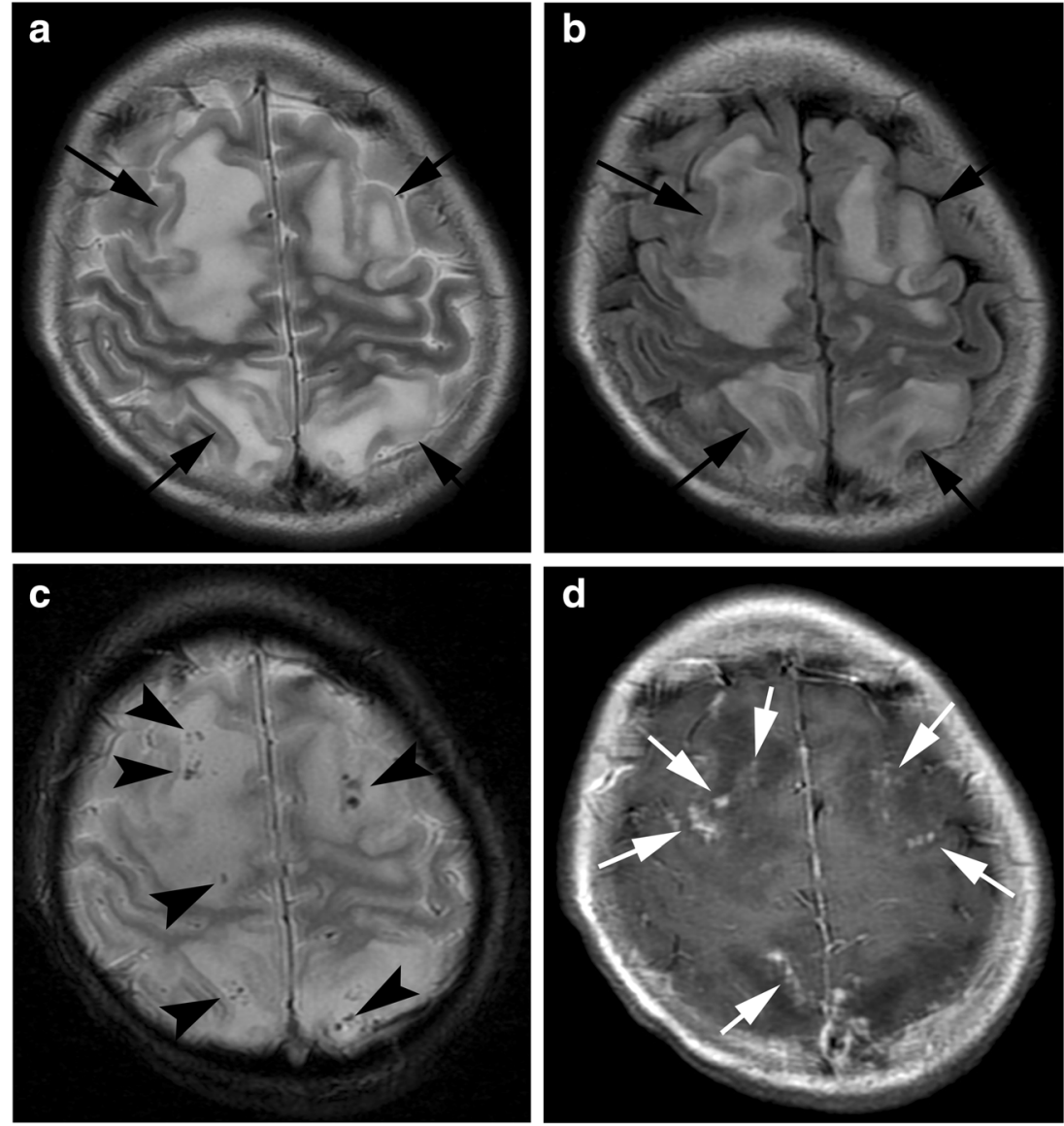

(Fig. 4), and it may progress to brain abscess if meningeal inflammation spreads to the brain parenchyma. In brain abscess, sudden onset headache and focal nervous disturbance like motor paralysis, convulsions, visual disturbance and cerebellar ataxia are observed. Progression of clinical symptoms in a matter of hours is characteristic for brain abscess. Fever is recognised only in about half of cases. MRI shows iso- or hyper-intensity on T1-weighted images with a low signal intensity rim on T2-weighted images. Post-contrast images show ring enhancement. Marked central diffusion restriction and rapid growth can help to differentiated abscess from neoplasm.

Treatment with broad-spectrum antibiotics with narrowing of coverage as possible is the general approach to treatment.
Fig. 4 Pyogenic meningitis. An 8-year-old boy with acute myeloid leukaemia, who had received chemotherapy including intensification treatment, presented with esotropia. a FLAIR image shows hyperintensities along the surface of the brain stem (arrows).

Communicating hydrocephalus is also seen (white arrows). b These lesions show enhancement (arrows)
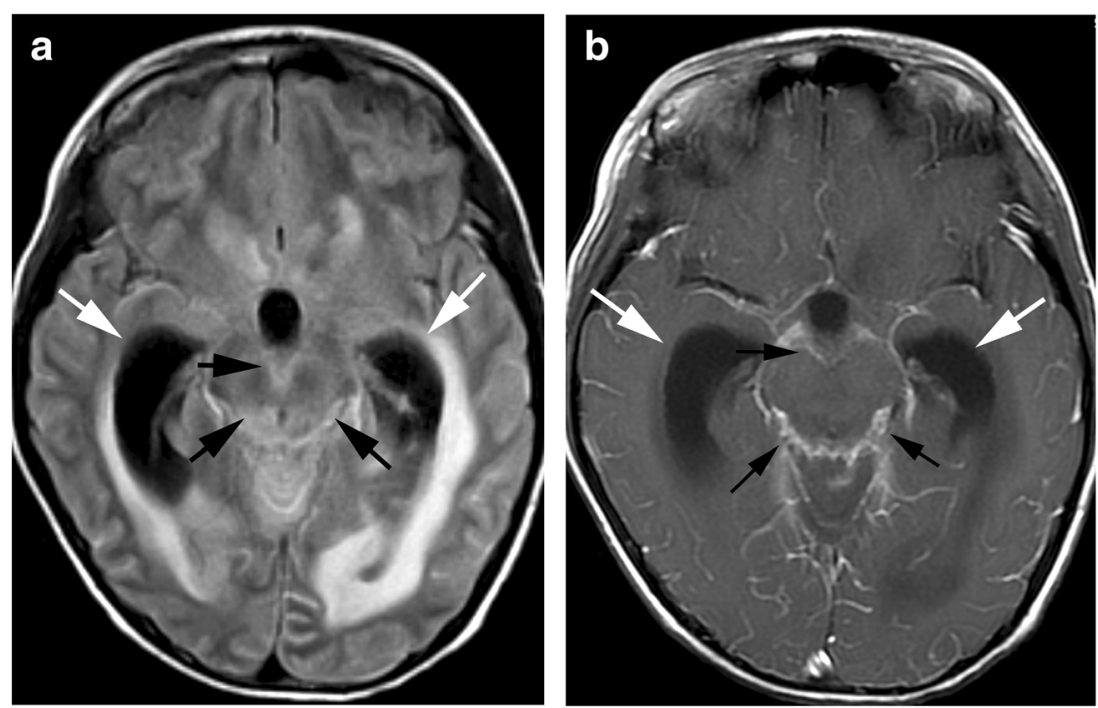
Viral infection The most common pathogen in viral infection in patients with haematological malignancy is the herpes virus. Among the herpes subtypes, HHV-6 is important as an opportunistic infection in immunosuppressed patients [25]. HHV-6 infection is present in $90 \%$ of children by 2 years of age. While inactive at sanctuary sites in the parotid gland and brain, HHV-6 activation leading to encephalitis occurs in immunosuppressed patients.

MRI findings of HHV-6 encephalitis are similar to acute disseminated encephalomyelitis, and scattered hyperintensities are seen in cerebral white matter on T2 weighted images [26].

Treatment is using with anti-viral agents such as acyclovir and gancyclovir, which are often started empirically when the imaging and clinical findings are suggestive.

\section{Treatment phase}

\section{Trousseau's syndrome (cancer-associated thrombosis)}

Trousseau's syndrome was first reported in 1865 by Armand Trousseau as a condition of cerebral infarction and pulmonary embolism due to multiple venous thrombosis associated with gastric cancer. In 1977, Sack et al. reported that Trousseau's syndrome is chronic disseminated intravascular coagulation (DIC) associated with non-bacterial thrombotic endocarditis and arterial thrombosis in patients with malignancy. Currently, the term "Trousseau's syndrome" is often used to describe a hypercoagulation disorder associated with various malignancies. It is reported that the risk of venous thromboembolism, including both deep vein thrombosis and pulmonary embolism, is fourfold to sevenfold higher in patients with cancer than those without cancer [27]. Neurological symptoms depend on the infarcted area; however, altered mental status and convulsion are often observed. Although Trousseau's syndrome is classified as a treatment phase complication in this article, it can occur as a primary symptom in some patients with paediatric cancer.

The brain is abundant with thromboplastin, which triggers the exogenous coagulation cascade and is thought to be a target of DIC due to the lack of thrombomodulin, a thrombin antagonist. Although the mechanism of hypercoagulability in patients with cancer has not been fully elucidated, it is thought that tumour cells express tissue factors that activate the coagulation cascade, including cellular procoagulants such as tumour procoagulant and factor $\mathrm{X}$ receptors. These lead to thrombosis by inducing cell-cell interactions with platelets, monocytes and endothelium via inflammatory cytokines, tumour antigens, and their immunoconjugates, which promote coagulation activation. Although malignant tumours that cause Trousseau's syndrome tend to be solid, children with any malignancy are at increased risk [28].

Certain MRI features can suggest this entity. Trousseau's syndrome should be considered, particularly when cerebral infarction involves three or more vascular territories. Microemboli are usually scattered in multiple vascular territories [29, 30]. In addition, MRI with MR venography is useful for the diagnosis of dural sinus thrombosis. MR venography can show loss of the flow void in an affected dural sinus [31] (Fig. 5). Since there are no clear diagnostic criteria for Trousseau's syndrome, it is important to search for malignant tumours when unexplained cerebral infarction or sinus thrombosis are found.

Regarding therapy, in addition to treating the underlying cancer, anticoagulant therapy using heparin is also usually necessary; warfarin is usually ineffective.

\section{Posterior reversible encephalopathy syndrome (PRES)}

PRES is an acute neurotoxic syndrome of characteristically reversible subcortical vasogenic brain oedema in patients with acute neurological symptoms. The pathophysiology of PRES is thought to be due to failure of cerebral blood flow autoregulation from endothelial dysfunction [32]. Predisposing conditions associated with PRES in patients with cancer include chemotherapeutics or immunosuppressant administration, infection and autoimmune disorders. Hypertension is associated with paediatric PRES, but because the cerebral blood flow autoregulation threshold is lower in children than in adults, the mean blood pressure at the onset of PRES is also lower [33]. The mean blood pressure at the onset of paediatric PRES was reported to be $140 / 85 \mathrm{mmHg}$ [34]. The spectrum of neurological features observed in patients with PRES includes headache, seizure, visual disturbances and nausea.

Although the subcortical white matter and cortex are often involved, distribution of abnormal imaging findings in PRES is classified into four patterns: holohemispheric watershed, superior frontal sulcus, dominant parietal-occipital and partial or asymmetric [35]. The basal ganglia, brain stem and cerebellum are also sometimes involved. MRI shows regions of high signal intensity on T2-weighted or FLAIR images. Restricted diffusion can be seen in $15-30 \%$ of cases, which is generally associated with irreversible change [32] (Fig. 6). Contrast enhancement is seen in about $20 \%$ of patients with PRES. Intraparechymal or subarachnoid haemorrhage around cortical or subcortical lesions is seen in $10-25 \%$ of cases. Intraparenchymal haemorrhage is often multifocal; however, mass effect is rare. Subarachnoid haemorrhage spares the basilar cisterns [32, 36]. Additionally, temporal lobe involvement, restricted diffusion on MRI, and associated multi-organ failure are more frequent in paediatric PRES compared with adults [37].

Treatment of PRES depends on its cause. Anti-epileptic medication may also be appropriate.

\section{Methotrexate neurotoxicity}

Abnormalities of the cerebral white matter are seen in some patients following treatment with chemotherapeutic agents. 
Fig. 5 Trousseau's syndrome. (dural sinus thrombosis). A 5year-old boy with acute lymphocytic leukaemia presented with seizure and altered mental status. a A T2*-weighted image shows hypointensity in the superior sagittal sinus (arrows). b MR venography shows signal loss in the superior sagittal sinus (white arrow)
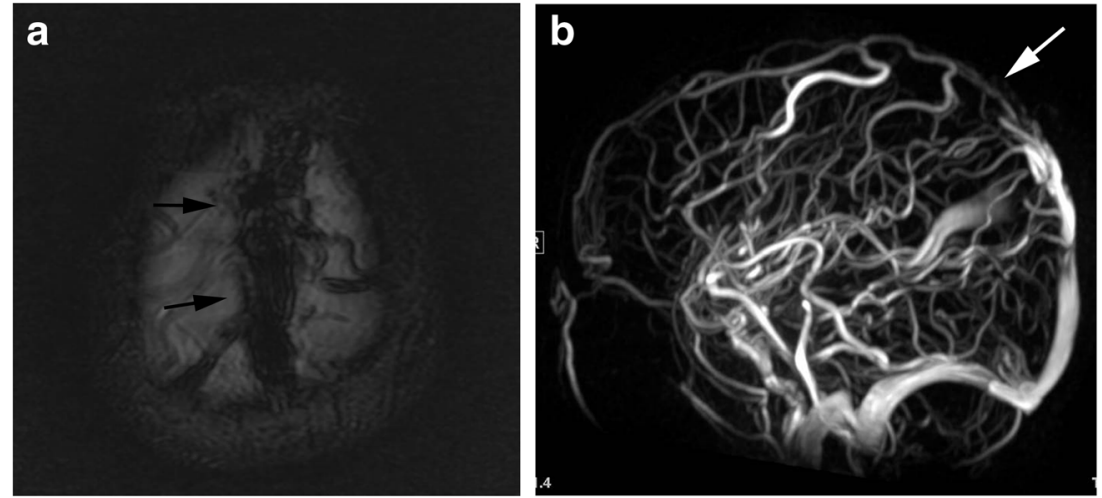

Although several drugs cause leukoencephalopathy, methotrexate (MTX) is the most common one in children. MTX is an antifolate drug used for treatment of diseases such as acute lymphoblastic leukaemia, malignant lymphoma and sarcoma. MTX neurotoxicity is found in 3-10\% of recipients.

It is believed that MTX can induce direct toxic effects to the CNS by damaging the neuronal tissue. Moreover, MTX interferes with the metabolic pathways of folate and induces biochemical alterations in excitatory amino acids, homocysteine, S-adenosylmethionine/S-adenosylhomocysteine, adenosine and biopterins [38], which can lead to neurological symptoms. High-dose intravenous administration, intrathecal administration, teenage and a history of radiation therapy are risk factors.

Its neurotoxicity can be classified as acute, subacute and chronic. Acute or subacute neurotoxicity can present with stroke-like symptoms such as aphasia, muscle weakness, sensory disturbance and ataxia, occurring within 2-14 days after initiation of MTX. Neurological symptoms are usually transient. In contrast, the chronic type can cause a slowly developing leukoencephalopathy and may progress to permanent impairment of neurological function.

MRI of acute neurotoxicity shows diffusion restriction on DWI in cerebral white matter, especially in the centrum semiovale or corona radiata, indicating intramyelinic oedema
(Fig. 7). Although T2-weighted and FLAIR images show hyperintensities, they may be quite subtle. DWI findings are normal after recovery, while T2 and FLAIR images usually show slight residual abnormalities. The cerebral cortex and cerebellum are also involved in atypical cases [39]. When imaging findings resembling cerebral infarction appear in patients receiving MTX, MTX neurotoxicity should be suspected.

After cessation of MTX and resolution of the associated neurotoxicity, subsequent intrathecal MTX administration is not associated with recurrence of MTX neurotoxicity.

\section{Post-remission phase}

\section{Hippocampal sclerosis}

Hippocampal sclerosis is a neuropathological condition with severe neuronal cell loss and gliosis in the hippocampus, specifically in the cornu ammonias area 1 and subiculum of the hippocampus. Although hippocampal sclerosis is generally associated with biphasic seizures of the infantile period, cerebritis, head trauma and perinatal brain injury, there are also some reports of hippocampal
Fig. 6 Posterior reversible encephalopathy syndrome. (PRES). A 5-year-old boy with acute lymphocytic leukaemia, who received bone marrow transplantation, developed lethargy during administration of tacrolimus. a A T2-weighted image shows hyperintensities in the occiptal lobes (arrows). b A susceptibility-weighted image (SWI) shows punctate signal loss in these lesions, suggestive of microhaemorrhage (arrowheads)
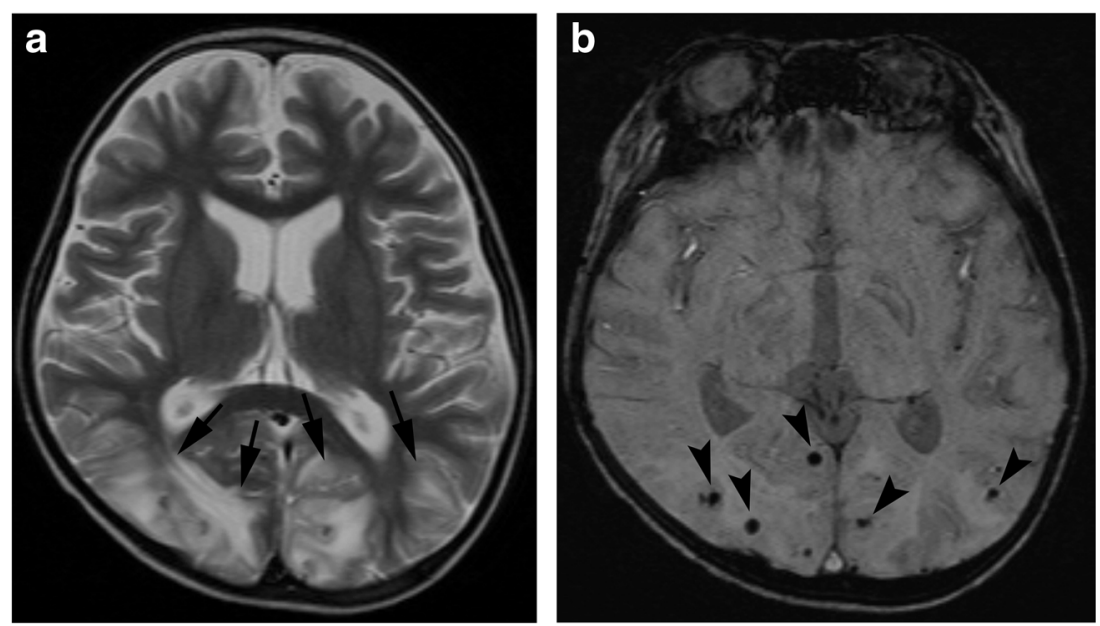
Fig. 7 Methotrexate neurotoxicity. (acute phase). A 14-year-old girl with acute lymphocytic leukaemia, who received intrathecal methotrexate, presented with left hemiplegia. a Diffusion-weighted image shows a small area of hyperintensity within the right centrum semiovale (arrow). b Apparent diffusion coefficient map shows restricted diffusion in this lesion (arrow)
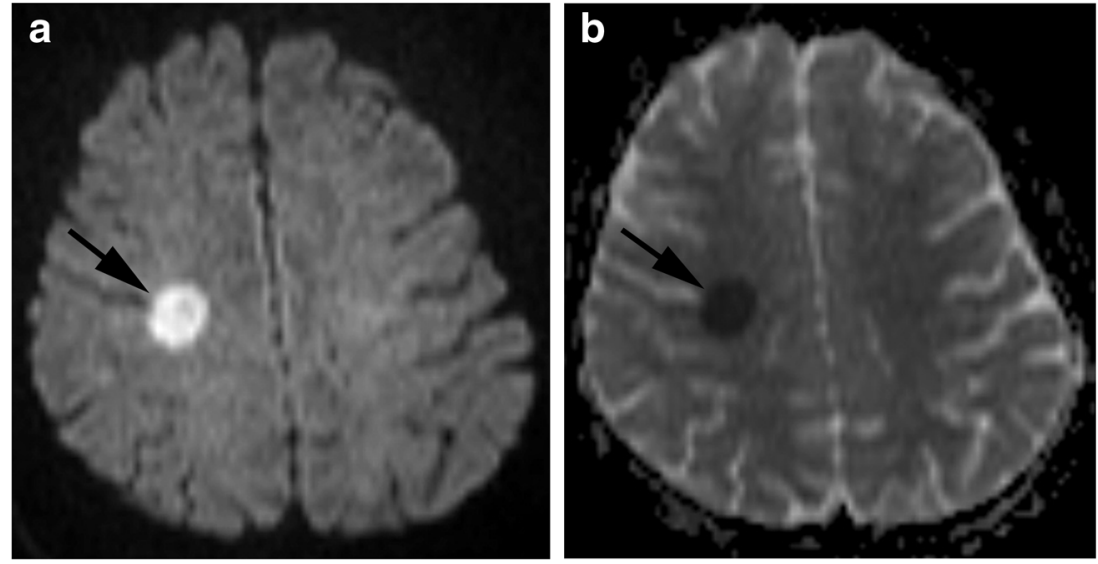

sclerosis in patients with haematological malignancy [40]. Various factors are thought to underlie the sclerosis seen in haematological malignancies, such as neurotoxicity due to methotrexate or cyclosporine, radiation exposure and bone marrow transplantation. Hippocampal sclerosis accounts for a large portion of refractory temporal lobe epilepsy in children. Although hippocampal sclerosis is highly associated with mesial temporal lobe epilepsy, it is not known whether hippocampal sclerosis causes temporal lobe epilepsy or temporal lobe epilepsy causes hippocampal sclerosis.

On MRI, coronal FLAIR and short tau inversion recovery images perpendicular to the hippocampus are useful. Atrophy and high signal intensity of the hippocampus and amygdala are observed (Fig. 8). In temporal lobe epilepsy, slight abnormal signal may be observed in the white matter at the temporal lobe tip, leading to the diagnosis of hippocampal sclerosis in the initial stages [41].

Medial temporal lobe epilepsy is usually refractory to anticonvulsant drugs; brain surgery is effective in such patients. Hence, early detection of hippocampal sclerosis in patients with haematological cancer is important, because these children are likely candidates for brain surgery.

\section{Radiation induced tumours}

Development of a secondary neoplasm is an important late complication of radiation therapy. Childhood cancer survivors who received cranial radiation therapy have an 8.1-52.3 times higher incidence of subsequent CNS neoplasms compared with the general population [42]. In adult patients with radiation induced brain tumours, meningiomas represent approximately $70 \%$, gliomas $20 \%$ and sarcomas $10 \%$ [43]. In paediatric populations, highgrade gliomas and meningiomas are the two most common subsequent CNS neoplasms, although medulloblastomas, primitive neuroectodermal tumours (PNETs), schwannomas and low-grade gliomas have also been reported [42]. Initial studies from the early 1990s had suggested that high-grade gliomas occur in the 1st decade after primary cancer diagnosis, but more recent studies with longer follow-up have shown that high-grade gliomas also occur in the 2nd decade after primary cancer therapy. Moreover, survivors of brain tumours who had not developed meningiomas at 20 years after diagnosis of their original cancer still had a $5.3 \%$ incidence of meningiomas in the subsequent decade [42]. Thus, deciding on an optimal screening regimen is challenging.

There is a recommendation that secondary brain tumour screening by MRI should be performed annually for the initial 5 years after completion of the radiotherapy, and thereafter repeated MRI screening should be performed only for the patients with neurological symptoms, such as headache, cognitive changes and seizures, particularly in those with a history of haematological malignancy or radiotherapy at a young age [44]. However, large sample, prospective randomised studies

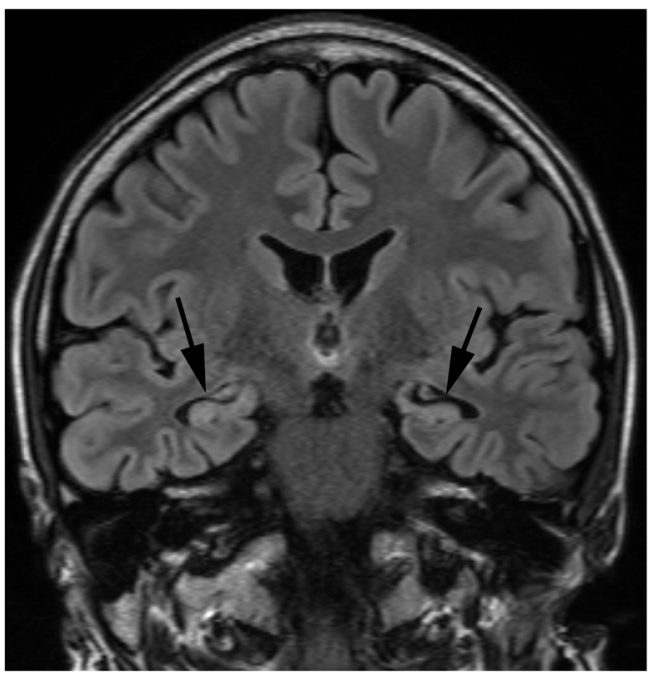

Fig. 8 Hippocampal sclerosis. A 24-year-old-man, who received bone marrow transplantation for malignant lymphoma at the age of six, presented with temporal lobe epilepsy. FLAIR image shows atrophy with high signal intensity in the bilateral amygdalae and hippocampi (arrows) 
Fig. 9 Radiation induced atypical teratoid/rhabdoid tumour. A 21year-old man, who received radiation therapy for optic glioma during infancy, presented with headache, appetite loss and somnolence. a A T2-weighted image shows a lobulated, solid mass with internal low signal intensity, indicating a small haemorrhage in the middle cranial fossa to basal ganglia (arrows). b Diffusion weighted image shows hyperintensity in the tumour (arrows). c Apparent diffusion coefficient map shows restricted diffusion in the tumour (arrows)
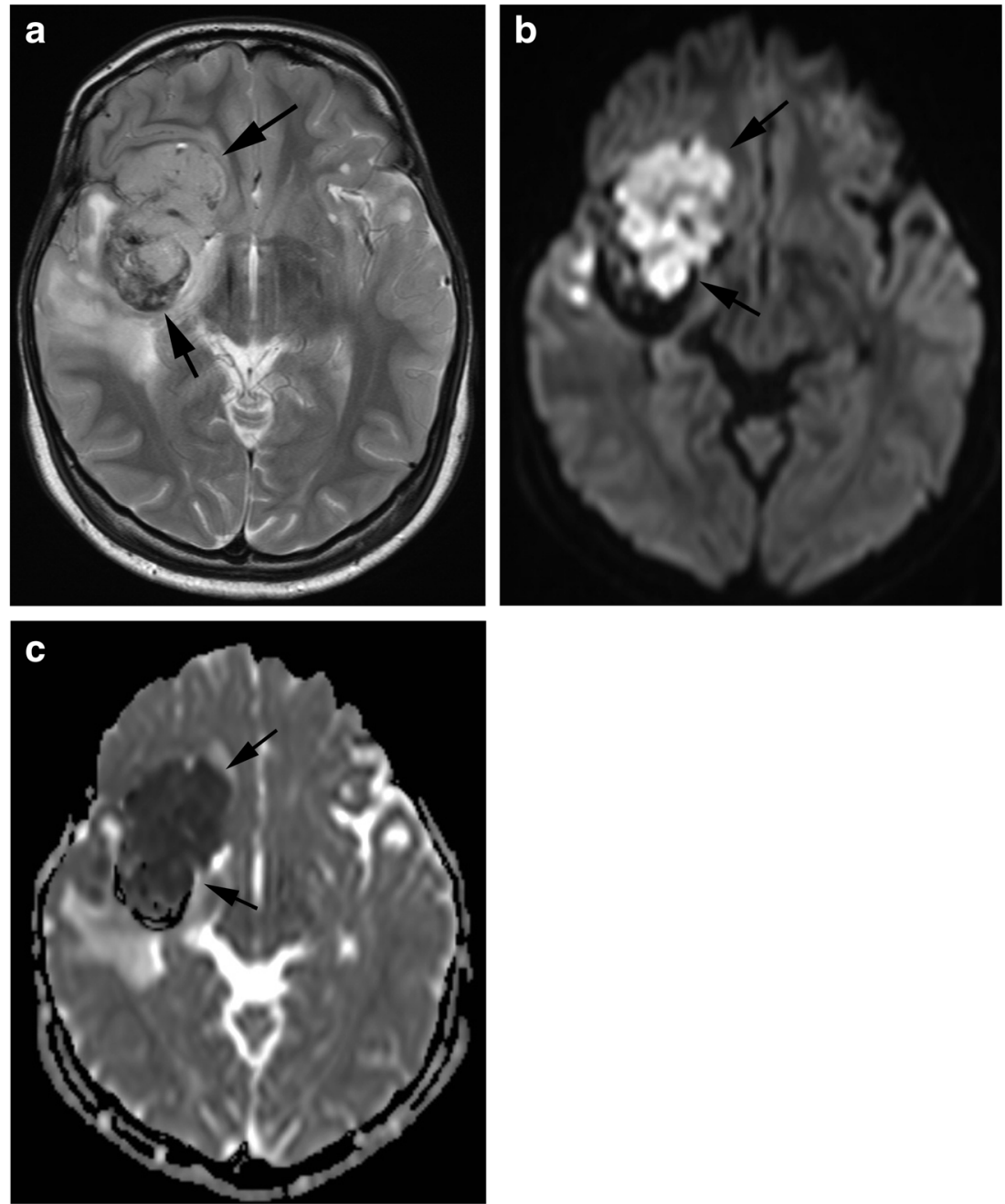

are needed in this regard. Radiation-induced gliomas are associated with high-grade gliomas in young people, multiplicity of gliomas and earlier age at presentation [45]. The neuroimaging appearance of radiation-induced tumours does not differ from that of other types (Fig. 9).

\section{Radiation induced focal haemosiderin deposition (RIFHD)}

RIFHD is a late complication of radiation therapy, which represents haemorrhagic or proliferative microangiopathies such
Fig. 10 Radiation induced focal haemosiderin deposition. A 29year-old woman, who received radiation therapy for acute lymphocytic leukaemia, had no symptom. a A T2*-weighted image shows focal haemosiderin deposition in the right insular and left temporo-parietal lobe (arrows). b FLAIR image shows slight low intensity area in the right insular (arrowheads). Left temporo-parietal small lesion is not apparent (arrow)
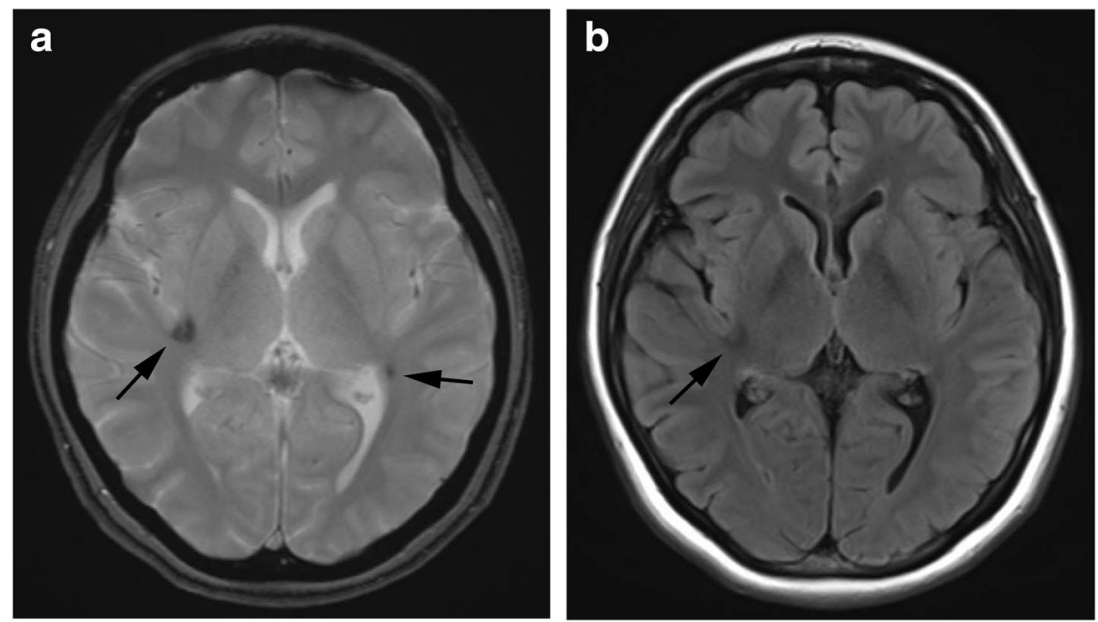
as capillary telangiectasias and cavernous malformations [46]. Pathologically, telangiectasias and cavernous malformations differ only in the presence or absence of intervening brain parenchyma among the dilated, thin-walled vascular channels. Because these similarities and transitional forms of these vascular malformations have been observed in some patients, radiation-induced telangiectasias and cavernous malformations have been proposed to exist along a spectrum driven by a common proliferative pathway [47].

The mechanism of RIFHD probably involves vascular injury, proliferation and dilation of vascular endothelium, hyalinisation and fibrinoid necrosis of blood vessel walls due to radiation therapy, and finally ischaemia and infarction due to narrowing of the vascular lumen [48]. The influence of age at the time of radiation therapy varies by report, and there is no consensus [46, $49,50]$. Radiation dose of 6-12 Gy has been reported as the minimum threshold level for development of RIFHD, and radiation dose positively correlates with frequency of RIFHD [50]. Although most patients with RIFHD are asymptomatic, symptomatic bleeding may occur. Infratentorial RIFHD is more prone to symptomatic bleeding compared with supratentorial RIFHD $[51,52]$. RIFHD is associated with neurocognitive dysfunction in primary brain tumour survivors [53].

On MRI, RIFHD shows mixed intensity with an enhancing cystic and/or solid component and an incomplete haemosiderin rim, which would be insufficient for a diagnosis of de novo cavernous malformation [54]. Small RIFHD lesions are best seen on iron-sensitive sequences such as gradient recalled echo imaging or susceptibility weighted imaging [48] (Fig. 10).

The treatment algorithm for RIFHD is not well established because the natural course of RIFHD has yet to be elucidated. Surgery may be considered in patients with repeated bleeding episodes and progressive neurological symptoms.

\section{Radiation induced white matter injury}

White matter injury is also an important late complication of radiotherapy. Radiation-induced white matter injury is divided into acute, early-delayed and late-delayed injury [55]. Acute and early-delayed reactions are often mild. Late-delayed injury is found as early as 3-4 months or as late as several years after completion of therapy.

It is believed to result mainly from permanent damage to blood vessels. Patients may develop progressive neurological symptoms. Pathologically, the affected white matter exhibits necrosis, with rarefaction and fragmentation of myelin and cellular disruption.

Imaging studies show variable patterns of injury, such as focal lesions or diffuse white matter abnormality. MRI demonstrates hypointensity on T1-weighted images and hyperintensity on T2-weighted images (Fig. 11). In whole-

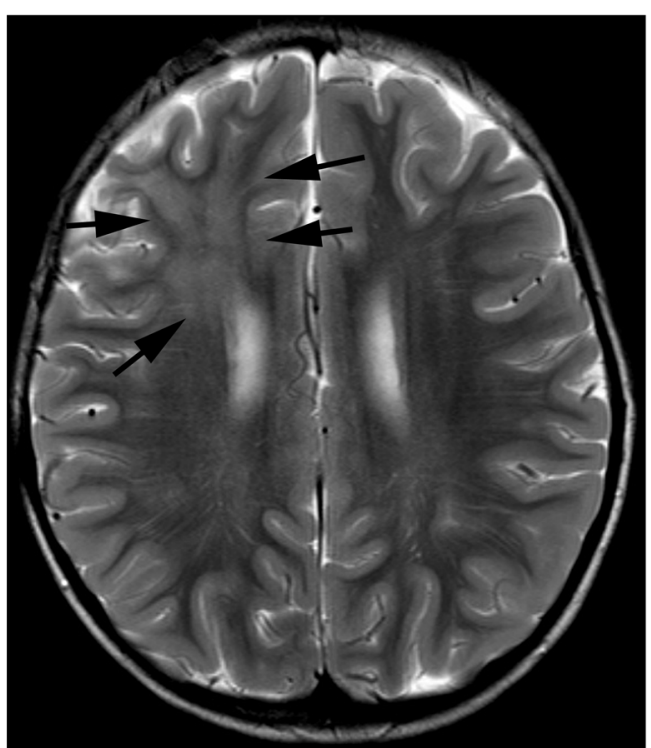

Fig. 11 Radiation induced white matter injury. A 5-year-old boy, who received radiation therapy for anaplastic ependymoma, was asymptomatic. A T2-weighted image shows hyperintensity in the right frontal lobe which was irradiated (arrows)

brain radiation, signal changes occur in the periventricular region and may progress in size and signal intensity over time, extending peripherally to the subcortical fibres [56]. Telencephalic commissural fibres are typically spared [56]. White matter volume loss may occur as a result of diffuse radiation injury [56]. Central necrosis within the lesions is uncommon in children [56].

\section{Conclusions}

Neurological complications of paediatric cancer are a substantial problem and are associated with significant morbidity and loss of quality of life for long-term survivors of paediatric cancer. It is critical for radiologists to recognise the imaging findings of these rare but important complications related to disease and treatment. MRI plays a significant role in the recognition and proper management of neurological complications of paediatric cancer.

\section{Compliance with ethical standards}

Conflict of interest We declare that we have no conflicts of interest.

Open Access This article is distributed under the terms of the Creative Commons Attribution 4.0 International License (http:// creativecommons.org/licenses/by/4.0/), which permits unrestricted use, distribution, and reproduction in any medium, provided you give appropriate credit to the original author(s) and the source, provide a link to the Creative Commons license, and indicate if changes were made. 


\section{References}

1. Chemaitilly W, Sklar CA (2010) Endocrine complications in longterm survivors of childhood cancers. Endocr Relat Cancer 17: R141-R159

2. Kaderali Z, Lamberti-Pasculli M, Rutka JT (2009) The changing epidemiology of paediatric brain tumours: a review from the Hospital for Sick Children. Childs Nerv Syst 25:787-793

3. Soussain C, Ricard D, Fike JR, Mazeron JJ, Psimaras D, Delattre JY (2009) CNS complications of radiotherapy and chemotherapy. Lancet 374:1639-1651

4. Wells EM, Dalmau J (2011) Paraneoplastic neurologic disorders in children. Curr Neurol Neurosci Rep 11:187-194

5. Alavi S (2013) Paraneoplastic neurologic syndromes in children: a review article. Iran J Child Neurol 7:6-14

6. Gultekin SH, Rosenfeld MR, Voltz R, Eichen J, Posner JB, Dalmau J (2000) Paraneoplastic limbic encephalitis: neurological symptoms, immunological findings and tumour association in 50 patients. Brain 123:1481-1494

7. Dalmau J, Gleichman AJ, Hughes EG et al (2008) Anti-NMDAreceptor encephalitis: case series and analysis of the effects of antibodies. Lancet Neurol 7:1091-1098

8. Waldenström J (1944) Incipient myelomatosis or 'essential' hyperglobulinemia with fibrinogenopenia a new syndrome? Acta Med Scand 117:217-246

9. Kong SG, Seo JH, Jun SE, Lee BK, Lim YT (2014) Childhood acute lymphoblastic leukemia with hyperleukocytosis at presentation. Blood Res 49:29-35

10. Park MS, Kim BC, Kim IK et al (2005) Cerebral infarction in IgG multiple myeloma with hyperviscosity. J Korean Med Sci 20:699-701

11. Algharras AA, Mamourian A, Coyne T, Mohan S (2013) Leukostasis in an adult with AML presenting as multiple high attenuation brain masses on CT. J Clin Diagn Res 7:3020-3022

12. Stone MJ, Bogen SA (2012) Evidence-based focused review of management of hyperviscosity syndrome. Blood 119:2205-2208

13. Kollias SS, Ball WS Jr, Tzika AA, Harris RE (1994) Familial erythrophagocytic lymphohistiocytosis: neuroradiologic evaluation with pathologic correlation. Radiology 192:743-754

14. Morimoto A, Nakazawa Y, Ishii E (2016) Hemophagocytic lymphohistiocytosis: pathogenesis, diagnosis, and management. Pediatr Int 58:817-825

15. Lehmberg K, Sprekels B, Nichols KE et al (2015) Malignancyassociated haemophagocytic lymphohistiocytosis in children and adolescents. Br J Haematol 170:539-549

16. Celkan T, Berrak S, Kazanci E et al (2009) Malignancy-associated hemophagocytic lymphohistiocytosis in pediatric cases: a multicenter study from Turkey. Turk J Pediatr 51:207-213

17. Chung TW (2007) CNS involvement in hemophagocytic lymphohistiocytosis: CT and MR findings. Korean J Radiol 8: $78-81$

18. Vázquez E, Lucaya J, Castellote A et al (2002) Neuroimaging in pediatric leukemia and lymphoma: differential diagnosis. Radiographics 22:1411-1428

19. Starkey J, Moritani T, Kirby P (2014) MRI of CNS fungal infections: review of aspergillosis to histoplasmosis and everything in between. Clin Neuroradiol 24:217-230

20. Gaviani P, Schwartz RB, Hedley-Whyte ET et al (2005) Diffusionweighted imaging of fungal cerebral infection. AJNR Am J Neuroradiol 26(5):1115-1121

21. Yamada K, Shrier DA, Rubio A et al (2002) Imaging findings in intracranial aspergillosis. Acad Radiol 9:163-171
22. Lai PH, Lin SM, Pan HB, Yang CF (1997) Disseminated miliary cerebral candidiasis. AJNR Am J Neuroradiol 1:1303-1306

23. Mora J, White M, Dunkel IJ (1998) Listeriosis in pediatric oncology patients. Cancer 83:817-820

24. Sakai C, Iuchi T, Ishii A, Kumagai K, Takagi T (2001) Bacillus cereus brain abscesses occurring in a severely neutropenic patient: successful treatment with antimicrobial agents, granulocyte colonystimulating factor and surgical drainage. Intern Med 40:654-657

25. Singh N, Carrigan DR (1996) Human herpesvirus-6 in transplantation: an emerging pathogen. Ann Intern Med 124:1065-1071

26. Kamei A, Ichinohe S, Onuma R, Hiraga S, Fujiwara T (1997) Acute disseminated demyelination due to primary human herpesvirus-6 infection. Eur J Pediatr 156:709-712

27. Stein PD, Beemath A, Meyers FA, Skaf E, Sanchez J, Olson RE (2006) Incidence of venous thromboembolism in patients hospitalized with cancer. Am J Med 119:60-68

28. Radulescu VC, D'Orazio JA (2017) Venous thromboembolic disease in children and adoslescents. In: Islam MS (ed) Thrombosis and embolism: from research to clinical practice. Springer International Publishing, Cham, pp 161

29. Finelli PF, Nouh A (2016) Three-territory DWI acute infarcts: diagnostic value in cancer-associated hypercoagulation stroke (Trousseau syndrome) AJNR Am J Neuroradiol 37:2033-2036

30. Schwarzbach CJ, Fatar M, Eisele P, Ebert AD, Hennerici MG, Szabo K (2015) DWI lesion patterns in cancer-related stroke-specifying the phenotype. Cerebrovasc Dis Extra 5:139-145

31. Vázquez E, Delgado I, Sánchez-Montañez A, Barber I, SánchezToledo J, Enríquez G (2011) Side effects of oncologic therapies in the pediatric central nervous system: update on neuroimaging findings. Radiographics 31:1123-1139

32. Fugate JE, Rabinstein AA (2015) Posterior reversible encephalopathy syndrome: clinical and radiological manifestations, pathophysiology, and outstanding questions. Lancet Neurol 14:914-925

33. Brady KM, Mytar JO, Lee JK et al (2010) Monitoring cerebral blood flow pressure autoregulation in pediatric patients during cardiac surgery. Stroke 41:1957-1962

34. Siebert E, Spors B, Bohner G, Endres M, Liman TG (2013) Posterior reversible encephalopathy syndrome in children: radiological and clinical findings - a retrospective analysis of a German tertiary care center. Eur J Paediatr Neurol 17: 169-175

35. Bartynski WS, Boardman JF (2007) Distinct imaging patterns and lesion distribution in posterior reversible encephalopathy syndrome. AJNR Am J Neuroradiol 28:1320-1327

36. Sharma A, Whitesell RT, Moran KJ (2010) Imaging pattern of intracranial hemorrhage in the setting of posterior reversible encephalopathy syndrome. Neuroradiology 52:855-863

37. Habetz K, Ramakrishnaiah R, Raina SK, Fitzgerald RT, Hinduja A (2016) Posterior reversible encephalopathy syndrome: a comparative study of pediatric versus adult patients. Pediatr Neurol 65:45-51

38. Vezmar S, Becker A, Bode U, Jaehde U (2003) Biochemical and clinical aspects of methotrexate neurotoxicity. Chemotherapy 49 : 92-104

39. Ziereisen F, Dan B, Azzi N, Ferster A, Damry N, Christophe C (2006) Reversible acute methotrexate leukoencephalopathy: atypical brain MR imaging features. Pediatr Radiol 36:205-212

40. Kasai-Yoshida E, Ogihara M, Ozawa M et al (2013) Temporal lobe epilepsy with hippocampal sclerosis in acute lymphoblastic leukemia. Pediatrics 132:e252-e256

41. Adachi Y, Yagishita A, Arai N (2006) White matter abnormalities in the anterior temporal lobe suggest the side of the seizure foci in temporal lobe epilepsy. Neuroradiology 48:460-464 
42. Bowers DC, Nathan PC, Constine L et al (2013) Subsequent neoplasms of the CNS among survivors of childhood cancer: a systematic review. Lancet Oncol 14:e321-e328

43. Kaschten B, Flandroy P, Reznik M, Hainaut H, Stevenaert A (1995) Radiation-induced gliosarcoma. Case report and review of the literature. J Neurosurg 83:154-162

44. Bilginer B, Türk CC, Narin F et al (2015) De novo formation of brain tumors in pediatric population following therapeutic cranial irradiation. Childs Nerv Syst 31:893-899

45. Pettorini BL, Park YS, Caldarelli M, Massimi L, Tamburrini G, Di Rocco C (2008) Radiation-induced brain tumours after central nervous system irradiation in childhood: a review. Childs Nerv Syst 24: 793-805

46. Yeom KW, Lober RM, Partap S et al (2013) Increased focal hemosiderin deposition in pediatric medulloblastoma patients receiving radiotherapy at a later age. J Neurosurg Pediatr 12:444-451

47. Rigamonti D, Johnson PC, Spetzler RF, Hadley MN, Drayer BP (1991) Cavernous malformations and capillary telangiectasia: a spectrum within a single pathological entity. Neurosurgery 28:60-64

48. Jain R, Robertson PL, Gandhi D, Gujar SK, Muraszko KM, Gebarski S (2005) Radiation-induced cavernomas of the brain. AJNR Am J Neuroradiol 26:1158-1162

49. Passos J, Nzwalo H, Valente M et al (2017) Microbleeds and cavernomas after radiotherapy for paediatric primary brain tumours. J Neurol Sci 372:413-416

50. Koike T, Yanagimachi N, Ishiguro $\mathrm{H}$ et al (2012) High incidence of radiation-induced cavernous hemangioma in long-term survivors who underwent hematopoietic stem cell transplantation with radiation therapy during childhood or adolescence. Biol Blood Marrow Transplant 18:1090-1098

51. Abla AA, Turner JD, Mitha AP, Lekovic G, Spetzler RF (2010) Surgical approaches to brainstem cavernous malformations. Neurosurg Focus 29:E8

52. Porter PJ, Willinsky RA, Harper W, Wallace MC (1997) Cerebral cavernous malformations: natural history and prognosis afterclinical deterioration with or without hemorrhage. J Neurosurg 87:190-197

53. Roddy E, Sear K, Felton E et al (2016) Presence of cerebral microbleeds is associated with worse executive function in pediatric brain tumor survivors. Neuro-Oncology 18:1548-1558

54. Cha YJ, Nahm JH, Ko JE et al (2015) Pathological evaluation of radiation-induced vascular lesions of the brain: distinct from De novo cavernous Hemagioma. Yousei Med J 56:1714-1720

55. Greene-Schloesser D, Robbins ME, Peiffer AM, Shaw EG, Wheeler KT, Chan MD (2012) Radiation-induced brain injury: a review. Front Oncol 2:73

56. Ball WS Jr, Prenger EC, Ballard ET (1992) Neurotoxicity of radio/ chemotherapy in children: pathologic and MR correlation. AJNR Am J Neuroradiol 13:761-776

\section{Publisher's Note}

Springer Nature remains neutral with regard to jurisdictional claims in published maps and institutional affiliations. 